\title{
Phonological processing in language production: time course of brain activity
}

\author{
St. Heim ${ }^{\text {CA }}$ and A. D. Friederici \\ Max Planck Institute of Cognitive Neuroscience, PO Box 500 355, 04303 Leipzig, Germany \\ ${ }^{\mathrm{CA} C o r r e s p o n d i n g ~ A u t h o r: ~ h e i m @ c n s . m p g . d e ~}$ \\ Received 3 June 2003; accepted 24 June 2003 \\ DOI: 10.1097/0I.wnr.0000091|33.75061.2d
}

\begin{abstract}
Recent neuroimaging studies provide evidence for a shared neural network for phonological processing in language production and comprehension. The temporal dynamics in this network during comprehension has been investigated by Thierry et al., who showed a primacy for Wernicke's over Broca's area. In the present study, we demonstrate the reversed pattern for language production.
\end{abstract}

These results can be interpreted with respect to the functionality of the different regions within the shared network, with Wernicke's area being the sound form store and Broca's area a processor necessary to extract relevant phonological information from that store. NeuroReport 14:2031-2033 (c) 2003 Lippincott Williams \& Wilkins.

Keywords: BOLD response; Broca's area; fMRI; Language comprehension; Language production; Phonology; Time course; Wernicke's area

\section{INTRODUCTION}

The time course of brain activation during the processing of phonological information has been the focus of research for a long time. A variety of measures (speech onset latencies, reaction times, event-related potentials (ERPs) and lateralised readiness potentials (LRPs) as well as their corresponding magnetic fields (MEG) were employed to determine the temporal relationship between phonological and other (e.g. semantic) processes during language production and comprehension [1-6]. There is also extensive effort in identifying the brain regions supporting these processes. According to the classical neurological model [7], there are two regions crucial for language processing. Language production is said to be supported by Broca's area (Brodmann's areas (BA) 44 and 45 in the left inferior frontal gyrus (IFG); see [8] for the careful investigation of the actual anatomical boundaries of this region, and [9] for the corresponding probability atlas). Language comprehension, on the other hand, is supposed to rely on Wernicke's area (i.e. the posterior portion of the left superior temporal gyrus (pSTG) and adjacent regions; see [10] for a detailed discussion of the literature about the anatomical definition of Wernicke's area, and [11,12] for recent probability atlases of this region).

For phonological processing, there is convincing evidence for a shared neural network for language production and comprehension, consisting of the superior portion of the left BA44 and BA22 in the left pSTG [13-17]. There is also evidence for the involvement of parts of the planum temporale in specific phonetic processing at least in language comprehension [18]. Still, only little is known about the temporal interplay of these regions during phonological processing. Using fMRI, Thierry et al. [19] investigated the time course of activation in Broca's and Wernicke's area during language comprehension. The authors were able to demonstrate that the BOLD (blood oxygen level dependent) signal reaches its maximum in Wernicke's area $\sim 3$ s earlier than in Broca's area. However, it cannot be excluded that this temporal delay is a general property of these regions and not specific for phonological processing during comprehension, since it is known that the temporal characteristics of the BOLD response differ among brain regions [20].

Evidence for the fact that the neural activity in Wernicke's area also precedes that in Broca's area during language production was provided by Kober et al. [21] by means of a MEG dipole analysis. In two tasks (silent naming, silent reading), the authors observed a temporal primacy for the activity in Wernicke's area. However, the authors explicitly stressed that three of the eight subjects showed the reverse pattern. Considering this fact and, more importantly, the nature of the task that implies not only phonological, but also semantic and orthographic processes, no final conclusion can be drawn about the temporal interplay of Broca's and Wernicke's area during phonological processing in language production.

We have, therefore, re-analysed our data [15] concerning the neural correlates of phonological processing during language production. In this analysis, the focus was on the temporal dynamics of the BOLD signal in the two central regions supporting phonological processing (superior portion of BA44, henceforth Broca's area; pSTG, henceforth Wernicke's area) as measured by two different phonological tasks. 
We will briefly describe the methods and results of the Heim et al. [15] study before reporting the results of the actual time-course analysis.

\section{MATERIALS AND METHODS}

In an event-related design, eight healthy subjects (age 24-29 years, three females; informed consent was obtained) saw pictures of real objects and performed a total of four tasks,

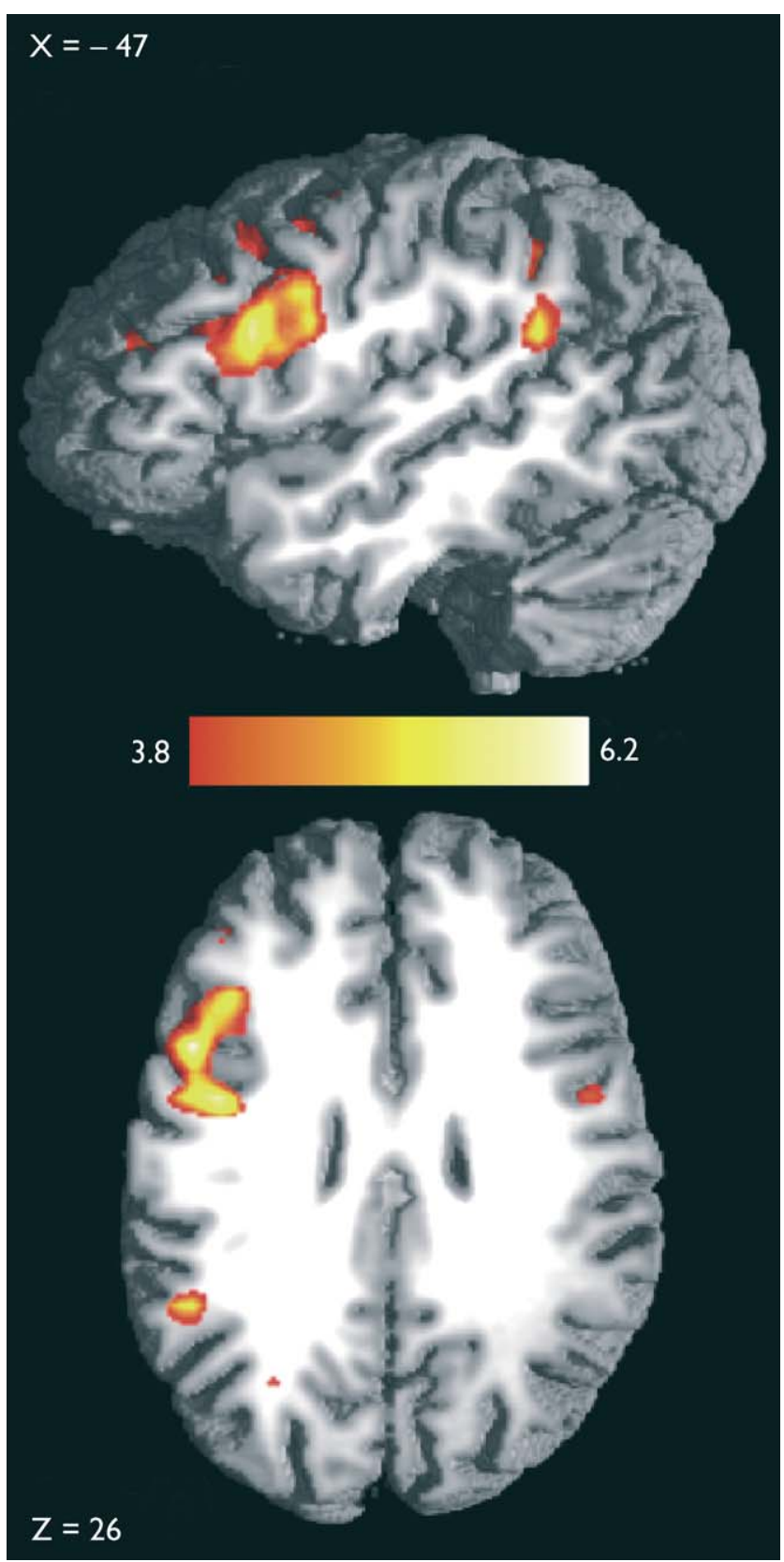

Fig. I. Statistical parametric maps (SPMz) of the activations in the conjunction analysis representing phonological processing, superimposed onto a high-resolution 3D MDEFT scan of a representative individual brain. The coloured scale bars indicate the activation strength. (For a complete list of activations see [15], Table 2). The displayed sections were taken at $\mathrm{x}=-47$ (sagittal) and $\mathrm{z}=26$ (axial) in Talairach coordinate space.
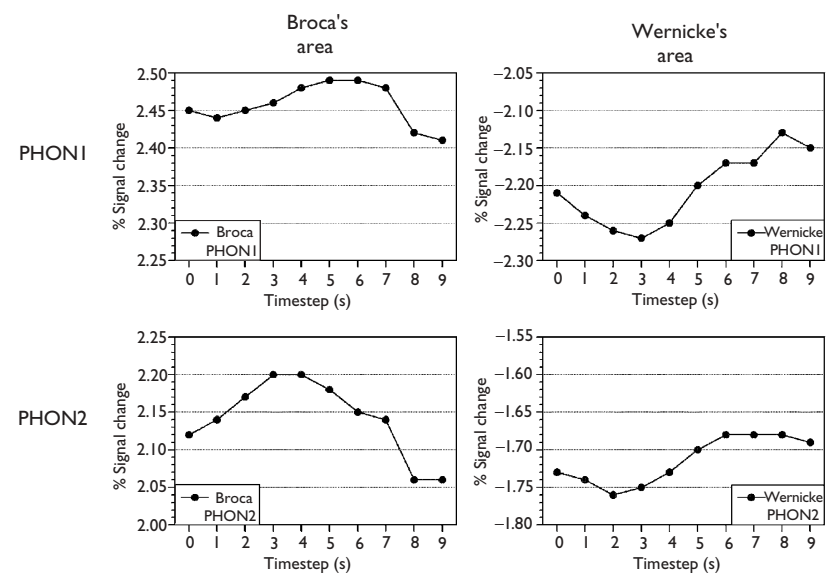

Fig. 2. Time courses of the modelled BOLD response in a $10 \mathrm{~s}$ time window as a function of region and task.

two phonological (PHON1 and PHON2), a semantic (SEM), and a baseline task (BASE). In PHON1, they indicated whether the German picture name started with the phoneme /b/ or not; in PHON2, they decided whether the initial phoneme was a vowel or not; in SEM, they pressed a button depending on whether the object was natural or man-made; and in BASE, they responded whenever a target picture appeared on the screen. Twelve axial slices $(6 \mathrm{~mm} / 2 \mathrm{~mm})$ were acquired using a $3 \mathrm{~T}$ scanner (Medspec 30/100, Bruker, Ettlingen) with a TR of $1 \mathrm{~s}$ and a FOV of $19.2 \mathrm{~cm}$ (for further details see the Methods section in [15]). The contrasts PHON1-SEM, PHON2-SEM, PHON1-BASE, and PHON2-BASE as well as conjunction analyses [22] were calculated. In all contrasts, there was activation in the superior portion of BA44 (Broca's area) and in the pSTG (Wernicke's area; Fig. 1). These results were comparable to those reported for phonological processing in language comprehension, indicating the existence of a common neural network for production and comprehension.

In the present analysis, we extracted the time course of the modelled BOLD response in Wernicke's and Broca's areas for both phonological conditions in a $10 \mathrm{~s}$ time window (Talairach coordinates PHON1: Broca -50,18,24; Wernicke -50,-54,29; PHON2: Broca -53,15,21; Wernicke $-53,-45,21)$. The data were taken from a 3D 26-voxel neighbourhood surrounding the voxel of peak activity (Fig. 2). Further, for each subject the time-to-peak (TTP) was calculated for each condition (PHON1, PHON2) and each region of interest (ROI). These data entered a $2 \times 2$ repeated-measures ANOVA with the factors condition and ROI. Missing values were replaced by the cell means.

\section{RESULTS}

There was a significant main effect for condition $(\mathrm{F}(1,7)=12.45 ; \quad p<0.001) \quad$ and $\quad \mathrm{ROI} \quad(\mathrm{F}(1,7)=49.51$; $p<0.001)$, and a significant interaction $(\mathrm{F}(1,7)=7.37$; $p<0.03)$. Post-hoc t-tests revealed that this interaction was due to a significant difference between the TTP in PHON1 and PHON2 in Broca's area $(t(7)=-5.82 ; p<0.001)$ and between the TTP in Broca's and Wernicke's area in PHON2 $(t(7)=-6.11 ; p<0.001)$. Overall, the TTP was shorter in 
Table I. Mean time-to-peak (TTP; ms) and standard deviation (in parentheses) as a function of region of interest (ROI) and condition.

\begin{tabular}{llc}
\hline & Broca's area & $\begin{array}{c}\text { Wernicke's } \\
\text { area }\end{array}$ \\
\hline PHONI & $5.78(1.45)$ & $7.00(1.5 \mathrm{I})$ \\
PHON2 & $3.00(0.54)$ & $7.00(1.5 \mathrm{l})$ \\
\hline
\end{tabular}

Broca's area than in Wernicke's area $(t(7)=-7.04 ; p<0.001$; see Table 1 for the absolute TTPs).

\section{DISCUSSION}

In the present study, we investigated the time course of brain activation in Broca's area and Wernicke's area induced by phonological processing during language production. There was an earlier time-to-peak (TTP) in Broca's than in Wernicke's area, and for the two phonological tasks (PHON1: /b/ or not?; PHON2: Vowel or not?), the TTP was shorter for PHON2 than for PHON1.

These results are interesting in the light of those obtained by Thierry et al. [19] in a language comprehension study, in two respects. First, whereas Thierry et al. observed a primacy for Wernicke's over Broca's area, the reversed pattern was found for production in the present study, with the mean temporal delay between the two peaks being identical $(\sim 3 \mathrm{~s})$. This result clearly speaks against the hypothesis of a general primacy for Wernicke's area that might have been supposed on the grounds of the reported studies $[19,21]$. Rather, the present data suggest that the cortical network for phonological processing is identical for production and comprehension with respect to the regions involved but characterised by a variable dynamics. In comprehension, Wernicke's area is activated first [19], and only if phonological segmentation is required, Broca's area is recruited [13]. In production, it appears that Broca's area is activated first in order to retrieve phonological information from the sound form store of the mental lexicon located in the pSTG [23].

Second, and supporting this notion, it appears that the overall TTP is longer for production than for comprehension (Thierry et al:: Wernicke $2.85 \mathrm{~s}$, Broca $5.61 \mathrm{~s}$; this study: Wernicke $7.00 \mathrm{~s}$, Broca $4.39 \mathrm{~s}$ ), although the difference between the TTP in comprehension and production is constant. Since both the present and the Thierry study used fMRI, this difference in the TTP is unlikely to be caused by the registration method per se. Rather, this pattern reflects the temporal sorting of the different stages in serial psycholinguistic models of language comprehension and production $[6,24]$. In these models, phonological processing takes place early in comprehension (before semantic processing) but late in production (after semantic processing). Thus, the temporal pattern of activation observed in the present study is in line with a large body of evidence from behavioural and electrophysiological data taken to support these serial models [2,4]. They add to these in identifying the different time courses for production and comprehension to be instantiated in the identical neural network.

\section{CONCLUSION}

In the present study, we were able to demonstrate that there is a variable temporal dynamics in the shared cortical network supporting phonological processing in language production and comprehension. This dynamics is such that Broca's area is activated before Wernicke's area in production, whereas the opposite pattern holds for comprehension.

\section{REFERENCES}

1. Jescheniak JD and Schriefers H. JEP LMC 24, 1256-1274 (1998).

2. Jescheniak JD, Schriefers H, Garrett MF and Friederici AD. J Cogn Neurosci 14, 951-964 (2002).

3. Levelt WJM, Praamstra P, Meyer AS et al. J Cogn Neurosci 10, 533-567 (1998).

4. van Turennout $\mathrm{M}$, Hagoort $\mathrm{P}$ and Brown CM. JEP LMC 23, 787-806 (1997).

5. Rodriguez-Fornells A, Schmitt BM, Kutas M and Münte TF. Neuropsychologia 40, 778-787 (2002).

6. Friederici AD. Trends Cogn Sci 6, 78-84 (2002).

7. Geschwind N. Science 170, 940-944 (1970).

8. Amunts K, Schleicher A, Bürgel U et al. J Comp Neurol 412, 319-341 (1999).

9. Mazziotta J, Toga A, Evans A et al. Phil Trans R Soc Biol Sci 356, 1293-1322 (2001).

10. Wise RJ, Scott SK, Blank SC et al. Brain 124, 83-95 (2001).

11. Rademacher J, Morosan P, Schormann T et al. Neuroimage 13, 669-683 (2001).

12. Westbury CF, Zatorre RJ and Evans AC. Cerebr Cortex 9, 392-405 (1999).

13. Burton MW, Small SL and Blumstein SE. J Cogn Neurosci 12, 679-690 (2000).

14. Démonet J-F, Chollet F, Ramsay S et al. Brain 115, 1753-1768 (1992).

15. Heim S, Opitz B, Müller K and Friederici AD. Brain Res Cogn Brain Res 16, 285-296 (2003)

16. Hickok G, Erhard P, Kassubek J et al. Neurosci Lett 287, 156-160 (2000).

17. Zatorre RJ, Evans AC, Meyer E and Gjedde A. Science 256, 846-849 (1992).

18. Jancke L, Wustenberg T, Scheich H and Heinze HJ. Neuroimage 15, 733-746 (2002).

19. Thierry G, Boulanouar K, Kherif F et al. Neuroreport 10, $2599-2603$ (1999).

20. Duann J-R, Jung T-P, Kuo W-J et al. Neuroimage 15, 823-835 (2002).

21. Kober H, Möller M, Nimsky C et al. Hum Brain Mapp 14, 236-250 (2001).

22. Price CJ and Friston KJ. Neuroimage 5, 261-270 (1997).

23. Hickok G and Poeppel D. Trends Cogn Sci 4, 131-138 (2000).

24. Levelt WJM, Roelofs A and Meyer AS. Behav Brain Sci 22, 1-75 (1999). 